\title{
LOS IMPACTOS DEL ANCLAJE TERRITORIAL EN EL MODELO DE NEGOCIOS: CASO EMPRESA ENO EN FRANCIA
}

\author{
ERIC PERSAIS \\ La Rochelle Business School, Francia \\ Grupo de Gestión Internacional Comparada (GIC) del laboratorio CEREGE \\ eric.persais@univ-poitiers.fr
}

RICARDO CUEVAS MORENO

\section{RESUMEN}

Este artículo tiene por objetivo estudiar el impacto del anclaje territorial sobre el modelo de negocios. Para ese propósito se utilizó el método de estudio de caso único, el cual permite comprender la riqueza del contenido de la actividad de una empresa francesa del sector de aparatos domésticos no eléctricos. El análisis está basado en el modelo recursos, competencias, oferta y valor (RCOV). Este pone en evidencia cómo la decisión de la empresa de anclarse en un territorio afecta esos aspectos, produciendo al mismo tiempo un aprendizaje organizacional. Así, la gerencia debe gestionar continuamente el equilibrio entre la eficiencia económica y las consecuencias extraeconómicas, cumpliendo simultáneamente su vocación de generar ganancia e impactar favorablemente el territorio que la alberga.

PALABRAS CLAVE: ANCLAJE TERRITORIAL, ESTUDIO DE CASO, MODELO DE NEGOCIOS, MODELO RCOV Y APRENDIZAJE ORGANIZACIONAL. Universidad La Salle, Cancún, México ricardo.cuevass@anahuac.mx

\section{ABSTRACT}

This article aims to study the impact of territorial anchorage on the business model. For this purpose, we use the method of single case study, which allows us to understand the richness of the content of the activity of a French company of electric domestic appliances sector. Our analysis is based on the model Resources, Skills, Offer and Value (RCOV). This demonstrates how the decision of the company's anchored in a territory affects these aspects, while producing an organizational learning. Thus, management must continually manage the balance between economic efficiency and extra-economic consequences, simultaneously fulfilling its objective to generate profit and favorably impact the territory that hosts it.

KEYWORDS: TERRITORIAL ANCHORAGE, CASE STUDIES, BUSINESS MODEL AND MODEL RCOV AND ORGANIZATIONAL LEARNING. 


\section{INTRODUCCIÓN}

Criticado durante mucho tiempo en el seno de la comunidad científica, el modelo de negocios (MN) es desde entonces objeto de un gran número de investigaciones en ciencias de la administración. Los límites precisos entre este concepto y el de estrategia por parte de los investigadores, contribuyen a establecer el MN como un tema guía en la literatura especializada. Si consideramos que el papel de la empresa consiste en generar riqueza para los accionistas que arriesgan invirtiendo su capital, entonces el MN se focaliza sobre un elemento clave de la administración.

Sin cuestionar esta visión de la empresa creadora de riqueza y potencialmente distribuidora de dividendos, la idea del valor creado tiende hoy a tomar un significado más amplio: no solamente la visión de valor centrado exclusivamente en la dimensión económica y financiera es demasiado restrictiva; la idea de centrar únicamente la problemática de la empresa en los accionistas aparece cada vez menos pertinente en relación con el poder del que disponen otros grupos de interés (stakeholders) sobre su funcionamiento. Por tal razón, se hace necesaria una visión más amplia del concepto de MN.

La demanda de una empresa social, más humana y en definitiva más preocupada por su impacto social y del cuidado al medio ambiente, conduce a algunos ejecutivos a afirmar el anclaje territorial de las empresas bajo su responsabilidad. Para ellos, la empresa tiene que participar en el esplendor del territorio donde se enmarca y prospera. No solo la creación de riqueza debe involucrar a todos los miembros de la sociedad (a fortiori, los grupos de interés). Así, la idea de crear riqueza debe ser tomada en un sentido mucho más amplio que su concepción generalmente asumida (valor económico / financiero). Por lo tanto, el anclaje territorial procede a la vez de la voluntad de extender el tema de la creación de valor a su dimensión no económica y de la afirmación de que los intereses de los grupos de interés (stakeholders) y los accionistas (shareholders) son inseparables y, por tanto, deben reconciliarse.

Esta visión "extendida" de un MN no es neutra, ya que tiene implicaciones importantes sobre este. A partir de ese supuesto, la formulación de nuestro problema se enuncia de la siguiente manera: ¿Cómo el anclaje territorial afecta a los diferentes aspectos del modelo de negocios (MN) de la empresa? Partiendo del modelo recursos, competencias, oferta y valor (RCOV) de Demil y Lecocq (2010), en el presente artículo se ofrece un análisis ciertamente limitado (ya que se basa en el caso de una empresa francesa que opta mayoritariamente por la fabricación nativa). Se muestra con claridad las repercusiones de la voluntad del anclaje territorial sobre los recursos y las capacidades de la empresa, sobre la organización de su cadena y su red de valor, y finalmente sobre su propuesta de valor en dirección a los grupos objetivo.

Este artículo está estructurado de la siguiente forma: en una primera parte, se aborda los aspectos teóricos y metodológicos de nuestra investigación y luego, en una segunda parte, se pone en evidencia las principales consecuencias de la visión extendida del MN en sus tres componentes. Finalmente, se expone las conclusiones y se abre las posibilidades para futuras investigaciones.

\section{ASPECTOS TEÓRICOS METODOLÓGI- COS DE LA INVESTIGACIÓN}

\section{El Modelo de Negocios (MN): definiciones y dimensiones}

La investigación en ciencias de la administración sobre el concepto de MN (modelo de negocios) tardó mucho tiempo. Varias razones son invocadas para explicar ese fenómeno (Lecocq, Demil y Warnier, 2006). Una de estas es que el MN es un concepto integrador de múltiples disciplinas 
de la administración. Esta transversalidad afecta necesariamente la difusión del concepto, pues la comunidad científica ha preferido durante mucho tiempo el modelo de divisiones funcionales. La segunda razón es que el concepto de MN nace a finales de los años noventa y esto coincide con el surgimiento de la "burbuja de Internet". La asimilación inadecuada del concepto por el comercio electrónico, paradójicamente frena de alguna manera su difusión a toda la comunidad científica, bien que el comercio en línea representa una parte importante del comercio mundial. Este también contribuyó al escepticismo por el MN; sus detractores subrayan brillantemente los peligros de tener un concepto aportado por el estudio abusivo del valor creado, provocado por el surgimiento de la "burbuja de Internet". La última razón se debe al hecho de que el concepto permaneció durante mucho tiempo falto de contenido y sufrió a la vez una profusión de definiciones que lo hacen parecer un fenómeno de moda, es decir, un concepto condenado a desaparecer. Existen varios enfoques sobre la concepción del MN pero la mayoría de los investigadores están de acuerdo en reconocer sus aportes, sobre todo porque propone un enfoque en el valor creado por la empresa en dirección a diversos grupos.

Moingeon y Lehmann-Ortega (2010) identifican tres concepciones del MN. El primero se centra en la apropiación de valor por la empresa. El MN se asimila al modelo de ingresos: "A business model is a model of doing business by which a company can sustain itself - that is, generate revenue. The essence of the idea is 'how you get paid' or 'how you make money' with a taxonomy of alternative mechanisms" (Chesbrough, Rosenbloom, 2002, p. 6-7). El segundo ofrece una perspectiva sobre cómo una empresa crea valor a través de procedimientos especiales. Por ejemplo, Tikkanen, Lamberg, Parvinen y Kallunki (2005, p. 792) establecen la siguiente definición:
We define the business model of a firm as a system manifested in the components and related materials and cognitive aspects. Key components of the business model include the company's network of relationship, operations embodied in the company's processes and resource base, and the finance and accounting concepts of the company.

La tercera definición se interesa no solamente a los modos operatorios, sino igualmente por la oferta hecha al cliente y los ingresos que se generan para la empresa. Por ejemplo, para Osterwalder, Pigneur y Tucci (2005, p. 5):

A business model is a conceptual tool containing a set of objects, concept and their relationships with the objective to express the business logic of a specific firm. Therefore, we must consider which concepts and relationships allow a simplified description and representation of what value is provided to customers, how this is done and with which financial consequences.

Esta tipología expresa finalmente la visión del MN cuyo ámbito se está expandiendo gradualmente. Al principio centradas en los resultados (creación de valor financiero), las definiciones tienden a incluir los elementos (componentes) del MN, así como los procesos que crean valor; luego, abordan este concepto desde una perspectiva sistémica estudiando la contribución del valor para los clientes y su impacto en los ingresos generados por la empresa (Stewart y Zhao, 2000). Obviamente, otros autores están interesados simplemente en la descripción del MN. Este es particularmente el caso de Osterwalder y Pigneur (2009), quienes proponen el famoso modelo CANVAS. Una virtud de este último estudio es que permite democratizar el concepto y promover su uso por los profesionales. 
Un número importante de autores tratan de describir las diferentes dimensiones del MN. Por ejemplo, Stähler (2002, p. 6) establece lo siguiente:

A business model (...) comprises of (1) A description what value a customer or a partner receives from the business. It's the value proposition, and it answers the question: what value the business creates for its stakeholders? (2) A description of the products and services the firm is providing. It answers the question: what does the firm sell? (3) A description of the architecture of value creation. It answers the question: how is the value in what configuration being created? (4) The value and sustainability of the business is being determined by its revenue model. It answers the question: with what do we earn money?

En ese mismo sentido, Moingeon y LehmannOrtega (2010) sintetizan los tres aspectos del MN:

- La proposición de valor: incluye el tipo de clientes o los segmentos del mercado al cual la empresa se dirige; el producto o el servicio propuesto por el cliente.

- La arquitectura de valor: comprende la cadena de valor interna de la empresa según la acepción de Porter (1985); la red de valor, es decir el conjunto de relaciones con los proveedores, socios, etc.

- La ecuación de ganancia: incorpora el valor obtenido por la empresa, explicando el volumen de ventas, la estructura de costos y el capital invertido, estos reflejados en la arquitectura de valor.

El modelo RCOV de Lecocq et al. (2006) es también muy citado. Según estos autores, la elección que una empresa efectúa para generar ingresos (es decir, su MN) cubre tres dimensiones principales:

- Los recursos y competencias movilizados para proponer una oferta.

- $\quad$ La oferta hecha a los clientes (en general).

- La organización interna de la empresa (cadena de valor) y de sus transacciones con sus socios externos (red de valor).

Según estos mismos autores, las decisiones tomadas determinan la estructura de los ingresos y la estructura de gastos de la empresa, que conducen a la noción de margen que indica si el MN es rentable.

\section{El modelo de negocios ( $\mathrm{MN}$ ): hacia la necesidad de extender la problemática de la creación de valor}

Aunque las perspectivas expuestas previamente abordan el tema del MN clarificando diferentes perspectivas, todas reposan esencialmente sobre una concepción económica y financiera del rendimiento de la empresa (Maucuer, 2013). La difusión del desarrollo sustentable (Brundtland, 1987) y la necesidad de la empresa de equilibrar las diferentes dimensiones de su resultado obligan, desde entonces, a considerar diferente el MN. Esa es esencialmente la idea defendida por Dahan, Doh, Oetzel y Yazijiet (2010, p. 340): "Research has traditionally identified the purpose of the $\mathrm{MN}$ as generation and delivery of economic value: here we extend the notion to argue MN can be viewed as generators of social value and that economic and social value creation can be mutually reinforcing". Así, para Zott y Amit (2010, p. 219) la creación de valor se debe comprender de manera extensiva. Esta concierne no solamente a la empresa y sus propietarios: 
"A business model is geared toward total value creation for all parties... The greater the total, the greater the focal firm's bargaining power, and the greater the amount of value it can appropriate". En consecuencia, si el propósito de la empresa es la remuneración de los accionistas, el logro de este objetivo requiere que la empresa cree valor para todos sus grupos de interés (stakeholders) (Porter y Kramer, 2011).

Vial (2008, p. 26) subraya del mismo modo esa necesidad de extender la problemática de la creación de valor (por ejemplo, no económico) y de sus beneficiarios (los grupos de interés):

Rather than a classical input-output approach to the enterprise, the author's definition of a business model includes and explicitely mentions internal as well as external stakeholders. It emphasizes the central role of value proposition, which, of course, relates to monetary value but can also be understood to include non-monetary value stemming from values of the company quality, tradition and ethics...

Realizando una reflexión prescriptiva, Dahan et al. (2010, p. 326) sostienen que la colaboración entre empresas multinacionales y las ONG puede permitir el surgimiento de nuevos MN en países en desarrollo: "In such cross-sector partnerships, parties contribute complementary capabilities along each stage of the value chain to develop products or services that neither could produce alone, creating and delivering value in novel ways while minimizing costs and risks".

Las investigaciones de Maucuer (2013) abordan también ese tema. El autor muestra en particular que las asociaciones entre Suez Environnement y ciertas ONG coadyuvan al surgimiento de nuevos MN centrados en las proposiciones de valor más preocupadas por las cuestiones ambientales y sociales. Para este autor, el MN se definiría de la siguiente manera: "Una configuración de elecciones intermedias entre la estrategia y las prácticas que influyen en cómo una organización crea y redistribuye valor económico y social" (Maucuer, 2013, p. 41). Sin poner realmente en tela de juicio el propósito de la empresa (es decir, la creación de valor para los accionistas), este autor plantea tanto la cuestión de la naturaleza del valor creado como la de los beneficiarios.

Yunus, Moingeon y Lehmann-Ortega (2010, p. 311) prolongan aún más esa perspectiva, pues ellos estudian el Social Business. Los autores subrayan las dificultades inherentes en el sistema actual, que dan prioridad a los aspectos económicos y financieros. El MN social tiene las mismas obligaciones que un MN tradicional: "[While] its primary purpose is to serve society, a social business has products, services, customers, markets, expenses and revenues like a "regular" enterprise... It is no-loss, no-dividend, self-sustaining company that repays its owners' investments". Por último, el MN aparece como innovador basado en una forma de equilibrio y reconocimiento del papel social de la empresa por parte de todos (los accionistas y los grupos de interés): "The value proposition and value constellation of the social business model must link all stakeholders, including shareholders who understand and accept its social mision". (Yunus et al., p.317) En suma, la distinción del MN al que aquí se refiere con respecto al MN "clásico" no es la creación de valor financiero y económico, sino la creación de valor social.

Si la especificidad de los negocios sociales (Social Business) conduce naturalmente a ampliar el tema de la creación de valor y su dimensión social, lo cierto es que la empresa "clásica" está impelida también a cuestionar su MN y desarrollarlo, teniendo en cuenta las necesidades tanto económicas como no económicas de todos sus grupos de interés (stakeholders). Se habla de la necesidad que tiene en la actualidad la empresa por alcanzar un MN sustentable, es decir, un MN basado en la creación y distribución equilibrada 
de valor económico, social y ambiental entre los grupos de interés.

Para luchar contra la globalización y el nomadismo económico, cuyos efectos son desastrosos tanto para las poblaciones como para el medio ambiente, cada vez más empresas quieren hacer valer su anclaje territorial (Zimmermann, 2005). Para una empresa, el concepto de territorio no es fácil de definir, sobre todo porque la escala de su acción varía en el espacio y en el tiempo. Por otra parte, como Zimmermann (2008) lo señala, el territorio debe considerarse solamente como un receptáculo (más o menos pasivo) de la actividad económica. Debe ser concebido como una construcción (geometría variable) generadora, creadora de los recursos productivos en una dinámica basada en la combinación de lógicas endógenas y exógenas de desarrollo. Empresas y territorios se retroalimentan.

EL ORSE (2006, p. 7) subraya territorio de la siguiente forma:

Una construcción compleja hecha de las relaciones entre empresas, instituciones, un entorno de servicio, un marco de vida: la acción de la empresa no tiene lugar en un vacío social y económico, pero está incrustada en estructuras económicas, instituciones sociales y culturales históricamente construidas.

Según Zimmermann (2008, p. 116-117), el territorio va más allá del concepto de espacio geográfico al cual se le asocia a menudo:

Este espacio es más que un telón de fondo para la coordinación de los agentes y de las actividades económicas, este no constituye de ninguna manera la esencia de esta coordinación... El territorio, desde el punto de vista económico, surge cuando hay una conjunción entre la proximidad geográfica y la proximidad organizada.
Así, el anclaje territorial nace de las interacciones e interdependencias entre las grandes empresas y sus regiones de acogida, en particular, sobre las cuales afirma el ORSE lo siguiente: "están, naturalmente, en el corazón del surgimiento económico de las regiones en las que operan. Ellas aportan capital, tecnologías, know-how (le savoir-faire) y el empleo, como condición necesaria para el territorio" (ORSE, 2006).

Cariou, Fournie y Wallet (2006, p. 3) consideran lo siguiente:

la problemática (...) del anclaje territorial no proviene de un proceso de acoplamiento entre las características de una empresa y las de un territorio, sino más bien de una dialéctica empresa-territorio al seno de la cual se construye la interacción potencialmente generadora de una dinámica común de la empresa y el territorio.

El territorio no es una condición estática. Es un sistema "en perpetua evolución como resultado de las modificaciones técnicas, de cambios de las relaciones de fuerza" (Le Gall y Bériot, 2013, p. 31). Se desprende que la relación entre la empresa y su territorio es susceptible de generar beneficios tanto para una como para el otro (Harribey y Cardebat, 2013): la primera no puede crecer en un territorio que le es hostil, el segundo puede brillar en la medida en que la empresa encuentre las condiciones favorables para su crecimiento. Todo se desarrolla para que las acciones de la empresa y su territorio converjan para lograr un "ganar-ganar".

Zimmermann (2008, p. 116) Ilama a este nivel "encuentro productivo", entendido de la siguiente forma:

la capacidad para aportar soluciones a ciertos problemas de producción inéditos, y esto en un marco principalmente territorial, es decir, aprovechando los efectos de proximidad geográficos; este, el encuentro pro- 
ductivo, emerge como un elemento clave de un proceso de coproducción de recursos, en la base de la construcción territorial.

Consecuencia de esta coproducción de recursos, los límites entre la empresa y el territorio devienen porosos y sus dinámicas de desarrollo están inevitablemente ligadas (Le Gall y Bériot, 2013, p. 31).

Desde un punto de vista comercial, los problemas del anclaje territorial son particularmente fuertes, sobre todo en un contexto de la responsabilidad social de la empresa (ORSE, 2006): problemas de reputación, de licencia para operar, de gestión y previsión de riesgos, de recursos humanos, de innovación y de mercado, en fin, de la eficacia del enfoque de desarrollo sustentable. Los medios para lograr esto son múltiples y, obviamente, las empresas se centran generalmente en un actuar transversal como lo muestra la figura 1.

La elección de la empresa de optar por un anclaje territorial de sus operaciones es un acto de gran alcance. Este tiene necesariamente consecuencias sobre su modelo de negocios y eso es, en efecto, lo que se quiere demostrar. Para ese propósito se presenta a continuación la metodología de nuestra investigación.

\section{METODOLOGÍA}

Como se mencionó en la primera parte de este artículo, se opta por el modelo de recursos, competencias, oferta y valor (RCOV) para evaluar los impactos del anclaje territorial sobre el MN de la empresa. Recuérdese que este contiene tres dimensiones (Lecocq et al., 2006):

Los recursos y las competencias: los RC se comprenden aquí en su capacidad para generar el valor. Estos se abordan no solo en una perspectiva estática (recursos propiedad, know-how desarrollado), sino también dinámica (cambios en el centro de competencias, capacidad de regeneración del MN).

- La oferta (O): por ejemplo, la propuesta de valor hecha por la empresa para los clientes y todos los grupos de interés: En esta perspectiva, el valor debe ser entendido

FIGURA 1

MEDIOS PARA LOGRAR EL ANCLAJE TERRITORIAL



Fuente: Elaboración propia. 
en sentido amplio y aprehenderse conceptualmente en sus diferentes aspectos: económico, social y medioambiental (de conformidad con el concepto de desarrollo sustentable).

- La organización de la cadena y de la red de valor (V): esto es establecer el posicionamiento de la empresa en este conjunto; en otras palabras, identificar las funciones que esta asumirá, y las que serán proporcionadas por sus socios.

A partir de estas dimensiones, se indagó cómo son impactadas por el anclaje territorial. No obstante, para cumplir ese objetivo e ilustrarlo, se eligió un estudio de caso único (Yin, 1990). A pesar de recibir críticas con frecuencia, este método es muy adecuado para el estudio de los fenómenos complejos, tales como el que se desarrolla en este artículo. Stake (1995) considera que un caso puede ser de interés instrumental y, por lo tanto, ser utilizado de forma objetiva para la comprensión de un fenómeno. Hadly-Rispal (2002, p.78) afirma lo siguiente: "Este tipo de estudio es apropiado en situaciones en las que el investigador quiere ilustrar fenómenos previamente definidos en un modelo teórico". Eso es, en efecto, lo que sucede en el presente caso. Además, este método permite comprender un fenómeno en el contexto, lo cual es particularmente importante con respecto a la empresa-objetivo, cuyo pasado explica, sin duda, la estrategia actual basada precisamente en esta noción de anclaje territorial. Es obvio que, aunque el estudio de caso puede tener un valor universal (Yin, 1990), esa no es la intención en este artículo. Inevitablemente es uno de los límites de esta investigación, cuyo alcance es analítico e ilustrativo.

La empresa ENO, sobre la cual se realiza el estudio de caso, forma parte del sector de fabricación de los aparatos domésticos no eléctricos. Situada en el departamento de Deux-Sèvres, la empresa nació en 1909 en Ardenas (Francia). Luego se instaló en la ciudad de Niort en 1915. Por no haber anticipado los cambios económicos durante los años 1980 estuvo a punto de desaparecer. Después de vivir un período de grandes turbulencias (1980-2000), la empresa fue adquirida por sus actuales propietarios en 2003. Anteriormente especializada en la calefacción de gas, sector en crisis, ENO se recuperó poniendo en práctica gradualmente una estrategia basada en la innovación. Luego, la dirigencia decidió retomar la producción de elementos de cocción para la navegación y al aire libre.

La empresa ENO emplea en la actualidad a un centenar de personas y realiza cerca de doce millones de euros en ventas (2013). Los productos que fabrica son parte de tres familias: los dispositivos de calefacción auxiliar, aparatos y sistemas de cocción para la navegación y, finalmente, planchas de cocina. Téngase en cuenta que, de esas tres familias, la primera y la tercera tienen una etiqueta territorial, es decir, la etiqueta Origine France Garantie (OFG). Los aparatos para actividades náuticas requieren la compra de acero inoxidable, que es imposible encontrar hoy en el mercado francés.

Las entrevistas para la recopilación de datos fueron realizadas en dos ocasiones: una en 2014 con una duración de dos horas; la otra en 2015 con una extensión de dos y treinta minutos (entrevistas personales). Los entrevistados fueron el presidente y el director general de la empresa. Para su realización se estableció una guía de entrevista elaborada en forma previa. Esta retoma naturalmente los diferentes aspectos del MN, a saber, los recursos y las competencias, la oferta (por ejemplo, la proposición del valor) y, en fin, la cadena y la red de valor de la empresa. El análisis de datos consiste esencialmente en un estudio temático. En seguida, se presentan los resultados de dicho análisis. 


\section{ANÁLISIS DE RESULTADOS: EL AN- CLAJE TERRITORIAL Y SUS IMPAC- TOS SOBRE EL MN}

En este apartado se expone cómo el anclaje territorial afecta la gestión de la empresa. Primero, se tomaron sucesivamente las diferentes dimensiones del $\mathrm{MN}(\mathrm{RC}, \mathrm{O}, \mathrm{V})$ y luego, se pusieron en evidencia las consecuencias de las decisiones estratégicas sobre cada uno de ellos. Tratándose del primer polo referente a los recursos y competencias, los dirigentes actuales decidieron desde un principio privilegiar la fabricación francesa. El hecho de que uno de los dirigentes sea un tecnólogo (ingeniero de formación) determinó en gran medida esa elección por parte de la empresa. La declaración del entrevistado es muy explícita al respecto: "Cuando eres industrial, estás orgulloso de fabricar en Francia". Sin embargo, dadas las implicaciones de costos, la elección de la fabricación francesa lleva a la empresa a optar por un posicionamiento de alta gama (PAG). Por lo tanto, la empresa es naturalmente conducida a gestionar tomando en cuenta los elementos de la competitividad de costos (calidad, flexibilidad e innovación), sin dejar de lado, por supuesto, la reducción de sus costos de producción. Es común que una empresa distinga cuatro tipos de recursos: inmateriales, materiales, financieros y humanos. A continuación, se esclarece en qué medida el anclaje territorial afecta a esos cuatro aspectos.

En cuanto a los recursos inmateriales, dos puntos merecen destacarse: el establecimiento de una política de marca y la necesidad de la empresa de obtener un reconocimiento por sus esfuerzos en su territorio mediante la obtención de una etiqueta: Origine France Garantie. Consecuencia directa de la elección de un anclaje territorial y de una fabricación de productos sobre todo franceses, la empresa optó por una estrategia de diferenciación a través de la calidad y la innovación (cf. supra). La nueva dirección decidió desde el comienzo crear una marca fuerte concebida como una promesa clara y exclusiva para el consumidor francés. Así, la empresa ENO desarrolló la marca Plancha Manía, símbolo, según sus dirigentes, de alto valor para el consumidor. Este valor, como se verá más adelante, supera el marco puramente comercial y llega a incidir sobre los aspectos simbólicos y de pertenencia, que son elementos por los cuales el consumidor toma un gran interés hoy.

Los esfuerzos de la empresa para participar en el resplandor de su territorio, en este caso Francia, representan, no obstante, un costo. Es por tal razón que algunas empresas se esfuerzan mediante la obtención de un signo distintivo que ponga en realce el carácter de la producción local, regional y nacional (por ejemplo, made in France). Por otro lado, ante la aparición de etiquetas, denominaciones de origen o marcas que no cumplen con las especificaciones, surge la necesidad de desarrollar una etiqueta territorial basada en un proceso real de certificación ${ }^{1}$. La etiqueta Origine France Garantie se creó en 2011 con el siguiente objetivo: "dar a los consumidores información clara y fiable sobre el origen de los productos y permitirá las empresas que realizan el proceso de certificación territorial, valorar mejorar su producción". Para obtener esa etiqueta, el producto debe cumplir con dos requisitosque se muestran en la figura 2.

En la actualidad, Origine France Garantie (OFG) es la única etiqueta territorial "verdadera" y es por eso que la empresa ENO decidió hacer certificar la mayor parte de sus productos cumpliendo con esas especificaciones. Esa etiqueta resulta importante pues deviene un argumento de venta, no solamente para el posicionamiento de alta gama (por ejemplo, gama plancha de cocción), sino

1. Un producto etiquetado made in France (producto por el cual $45 \%$ del valor agregado es producido en Francia) puede ser objeto de una veríficación por la aduana o la Dirección General de la Competencía, del Consumo y de la Represión de Fraudes (Direction Générale de la Concurrence, de la Consommation et de la Répression des Fraudes). Esto se debe a que esa etiqueta no está sujeta a ningún proceso de certificación. 


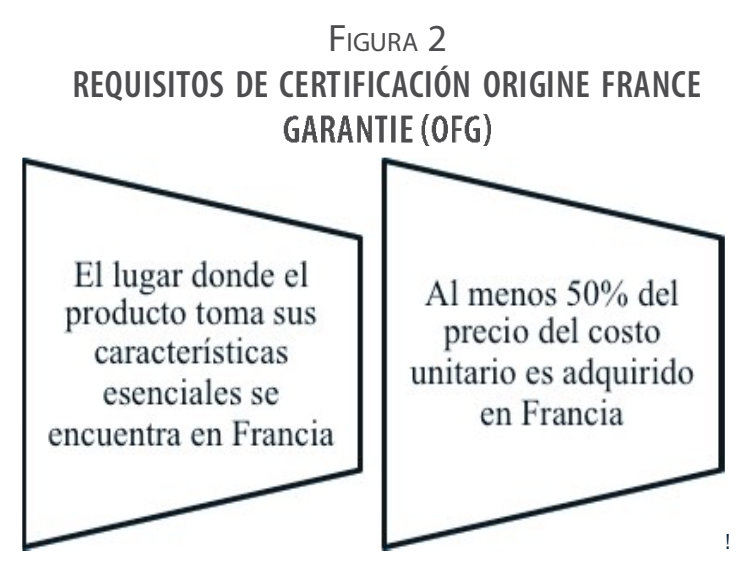

Fuente: Elaboración propia.

también para los productos de uso diario (por ejemplo, calefacción eléctrica o de gas). Según afirman los dirigentes de la empresa: "Para las planchas de cocina, el OFG es esencial porque se quiere venderlas caras para mantener nuestros márgenes de ganancia, así como los de nuestros distribuidores, a quienes debemos convencer de vender nuestros productos".

Los recursos materiales también se ven afectados por este deseo de establecer un anclaje territorial. La cuestión de costos de producción es evidentemente crucial, sobre todo para las empresas confrontadas a la competencia china, como es el caso de ENO, que fabrica bienes de equipo. En cuanto a los productos de consumo masivo (calentadores de gas), la empresa no puede permitirse el lujo de invertir en equipos más eficientes. De hecho, el retorno de la inversión no está garantizado, tratándose además de una actividad en el fin de su ciclo de vida: "Este es ur mercado que está declinando desde hace diez años; la disminución en términos de precios de venta, margen de ganancia...". El único apoyo de la empresa para mejorar los procesos de producción es a través de técnicas Lean Management, como lo destaca uno sus dirigentes:
En la industria, hay actividades que crean valor, otras que son un costo. El problema es que no podemos contratar, despedir... Esa es una actividad (la calefacción de gas) que ayuda a reducir la facturación; alguien que tuviera una gestión puramente financiera de la empresa la detendría instantáneamente...

Para los productos de alto valor agregado, se requiere que la empresa trabaje sobre los costos y los elementos de la competitividad de aquellos (calidad, flexibilidad). El producto estrella de la empresa, en este caso la plancha de cocción, requiere la aplicación de una técnica particular, que es la del esmaltado del hierro fundido. Hasta 2014, esta parte del proceso de producción, que representa el mayor valor agregado, se realizó de manera artesanal. En 2013, la empresa decidió invertir en dos robots de aplicación de pintura y en un horno de cocción automática. Estas inversiones permiten que la empresa sea mucho más flexible que antes. Además, se asegura la calidad de fabricación óptimo 2, que es un elemento clave para un producto que tiene como objetivo un alto nivel de posicionamiento. Por último, el impacto en los costos de producción es muy favorable para la empresa, ya que los costos de mano de obra son claramente menores que antes en virtud del cambio en su composición técnica de capital por la innovación (Marx, 1965).

El impacto del anclaje territorial sobre los recursos financieros es también significativo. Se observa, además, un movimiento inverso doble. Por un lado, la necesidad de que una parte importante del valor agregado se realice en suelo francés implica automatizar un gran segmento del proceso de producción (para cubrir, en particular, el costo de la mano de obra en Francia). Aunque el factor costo de producción no es el único elemento en juego (cf. supra), las inversiones necesarias para ello requieren un capital significativo. La flexibilidad del equipo de producción (hecho 
posible mediante la sustitución de las máquinas convencionales por las máquinas de comando numérico) engendrada por esta inversión permite reducir significativamente los requerimientos de fondos revolventes. De hecho, con respecto a la gama plancha, de una demanda estacional, la empresa debe fabricar previamente sus productos, en particular planchas de cocción, durante los períodos de punta y almacenar en previsión para responder a la fuerte demanda al principio del año (primavera y verano). En el caso de ENO, la compra de robots y el horno de esmaltado se financian en gran parte por la reducción de las existencias. En otras palabras, el impacto del anclaje territorial sobre los recursos financieros es casi neutro.

El impacto del enfoque de anclaje territorial sobre los recursos humanos es, por su parte, notable. El abandono de las tecnologías convencionales (por ejemplo, la aplicación manual de pintura de esmaltado) por las nuevas tecnologías (robots de esmaltado de pintura) requiere una revisión de la política de recursos humanos. Las habilidades requeridas son ahora diferentes: el hacer manual se desvanece mientras que la capacidad de pilotear una máquina numérica deviene una necesidad para la mayoría de los trabajadores (en particular los recién contratados). En este caso, la empresa ENO se beneficia de la aplicación de esa inversión para operar una renovación técnica y una renovación de su personal de producción. Dicho esto, esta transición se realiza gradualmente; se requiere una fase de adaptación progresiva y la implementación de una gestión provisional de empleos y competencias dentro de la empresa, como se observa a continuación.

La observación precedente nos permite abordar la cuestión de las capacidades relacionadas con la voluntad de la empresa para promover un anclaje territorial de sus actividades. ENO desarrolla durante ese proceso dos tipos de competencias, una en el campo de esmaltado y otra en el de diseño de productos innovadores dedicados a la cocina al aire libre. Sobre el primer punto, la empresa cuenta con experiencia (know-how o le savoir-faire) reconocida en el campo del esmalte de los alimentos sobre hierro fundido, ya que posee una etiqueta Enterprise du Patrimoine Vivant de esa técnica desde el año 2011. El abandono de la técnica artesanal de aplicación manual de la pintura en aerosol por la robótica no afecta el know-how de la empresa. Al contrario, permite mejorar el nivel de calidad del producto y dirigir a la empresa hacia un enfoque de calidad total.

Nótese aquí que la técnica de esmaltado desapareció durante cierto tiempo de la empresa y fue reactivada por el lanzamiento de la gama plancha. Uno de los dirigentes relata las dificultades para revivir ciertos know-how técnicos e industriales:

Hay muchos trabajos que se aprenden dentro de la empresa y que no se enseñan en las escuelas. (...) Fue muy difícil para nosotros, fue necesario reparar el horno, pero el know-how todavía existía, pero una vez que este desaparece, desaparece sin remedio. Así que la cuestión es preservar o reactivar el know-how (le savoir-faire).

Es interesante abordar, a través del esmaltado (en particular la aplicación de la pintura) la cuestión del aprendizaje organizacional. El caso ENO es una correcta ilustración de los modos de producción de conocimientos descritos por Nonaka (1994). Por ejemplo, este es el paso del conocimiento tácito al conocimiento explícito: el conocimiento personal (es decir, la habilidad del movimiento de mano desarrollado por los operadores asignados a esta tarea) se formaliza, se explica para poder ser retranscrito a los robots para la nueva forma de aplicación. Según Nonaka, esta externalización es el resultado de la interacción de lo tácito y lo explícito, es generadora de una poderosa creación de conocimiento organizacional. Es este proceso, sin duda, el que pone en práctica la empresa ENO al realizar esta 
transformación técnico administrativa en el contexto de anclaje territorial.

Sobre el segundo punto, el posicionamiento alta gama (PAG) y la voluntad de diferenciarse de sus principales competidores (en particular los fabricantes chinos) Ilevaron a la empresa a desarrollar un verdadero know-how en la innovación de productos. ENO es especialmente la primera empresa en ofrecer una plancha de cocción eléctrica (gama Electra, cuya calidad recibió un premio en la Feria de París en 2012) o lanzar una gama de planchas de cocción especial para el equipamiento de los barcos para actividades náuticas en 2013. Este know-how en la innovación de productos también fue galardonado en 2011 por OSEO Excellence como una de las dos mil empresas francesas más innovadoras. Su próximo reto es conseguir que la plancha de cocción forme parte de la cocina y de hacerla una parte esencial del hogar, tanto como el horno o la estufa.

Obsérvese ahora el segundo elemento del modelo RCOV, que se refiere a la oferta y, por lo tanto, a la propuesta de valor de la empresa. Obviamente, los primeros involucrados son los clientes, que contribuyen o no a través de sus compras al equilibrio del modelo económico. Es útil aquí disociar los tres dominios de actividad estratégica, que son la plancha de cocción, la calefacción y el equipamiento para barcos recreativos; el contexto es diferente en cada caso. Para los dos primeros, la empresa trabaja en B (to B) to C. ENO es directamente confrontada con la demanda de los clientes finales. Como lo señala uno sus líderes, el enfoque OFG iniciado por la empresa no era realmente una solicitud por parte de clientes:

Se tomó conciencia del hecho de que gobierna la desindustrialización en Francia y de la necesidad de salvar el know-how. Este es el origen de la etiqueta OFG. Nosotros aceptamos la apuesta. De principio, cuando hablamos de la etiqueta, era realmente un lenguaje de sordos para la gran distribución, los centros comerciales. El hecho es que hay cada vez más empresas que solicitan etiquetas, las grandes empresas (por ejemplo, Toyota, Peugeot; A. Montebourg tomó la marca de fabriqué en France). Hubo una toma de conciencia general, que tuvo un efecto de bola de nieve.

En la propuesta de valor, además de la cuestión de la utilidad en el sentido económico (valor instrumental), tres elementos parecen importantes en la actualidad en el comportamiento del consumidor: las dimensiones simbólica, económica/ social y cualitativa. En primer lugar, en el momento de las reubicaciones, el nomadismo económico y la presencia de empresas que operan en una perspectiva global, los clientes reconocen la preferencia por el hecho de que algunas empresas hayan resistido ese movimiento profundo, haciendo de su anclaje territorial un elemento clave de su estrategia. Así lo destaca uno de los dirigentes de ENO: "A nuestros clientes, nosotros les contamos una historia, les hablamos de un territorio próximo a ellos, de sus preocupaciones... Nosotros pensamos que esa es, en última instancia, toda la dimensión simbólica en la compra del producto etiquetado OFG".

En segundo lugar, los clientes son cada vez más conscientes de los efectos de sus compras en la economía del territorio. Ciertamente, un producto fabricado en un país de bajo costo de mano de obra es más barato, pero ese hecho implica la destrucción de puestos de trabajo industriales en el suelo francés. Corresponde a la empresa valorar los esfuerzos realizados para el desarrollo de su territorio. Desde ese punto de vista, la etiqueta OFG es una garantía de que el producto es realizado principalmente en Francia. Dicho esto, su reputación no es suficiente hoy. Los clientes no siempre ven los pros y los contras del anclaje territorial. Así lo subraya uno de los líderes de ENO: 
A los clientes se les debe explicar la etiqueta. Es una realidad. Ahora, cuando se les presentan las implicaciones de tener trabajadores en Francia, el compromiso social (por ejemplo, empleamos una treintena de personas discapacitadas), ellos se adhieren. Los clientes están dispuestos a pagar un poco más caro, por la distribución. Los clientes son sensibles, de mente abierta.

Por último, está el aspecto cualitativo de la compra. Para un comprador francés o extranjero, el hecho de que un producto sea fabricado mayoritariamente en Francia es sinónimo de garantía de calidad: "La gente dice que compra sus productos porque están hechos en Francia, es buena calidad..." En otras palabras, el alto estándar de fabricación francesa es en sí mismo una garantía de calidad del producto para los clientes.

La compañía ENO también trabaja B to C, especialmente en el mercado del equipamiento para barcos de actividades náuticas. Proporciona a varios fabricantes de embarcaciones náuticas, en su mayoría franceses, electrodomésticos de cocina a bordo. De acuerdo con los dirigentes de la compañía, dos criterios son relevantes en este mercado: en primer lugar, la calidad del producto, junto con la proximidad geográfica; y en segundo lugar, el establecimiento de relaciones de asociación con sus proveedores. Para esos productos, ENO no detenta la etiqueta OFG, sobre todo porque estos tienen como su característica principal utilizar como materia prima el acero inoxidable, que no se fabrica en suelo francés. Pero, señala uno de los dirigentes:

Tenemos menos interés en que esta gama sea etiquetada... Hacemos muy poco B to C (mercado de repuestos)... Este se instala principalmente con los fabricantes de barcos que nos compran directamente. Ellos, que saben dónde fabricamos, son un nicho de mercado... El reto es la exigencia que hay alrededor de la etiqueta. A los fabricantes de embarcaciones no tenemos necesidad de convencerlos, ellos saben quiénes somos. No es lo mismo con nuestros clientes privados...

Así, aunque el producto sea etiquetado OFG, tomando en cuenta el primer criterio (característica principal en Francia), se debe aceptar la idea de que el anclaje territorial juega un papel importante en la oferta del producto. Los clientes parecen estar seguros por la idea de que su proveedor de equipos de cocina a bordo es francés, que está próximo a ellos (oeste de Francia) y que las relaciones establecidas con su proveedor sean de asociación. En suma, nuestro análisis muestra que, aparte de estos aspectos cualitativos, no hay otros criterios (económicos, sociales o simbólicos) que entren en juego en las compras en B to B. En otras palabras, solamente cuenta el aspecto profesional en las relaciones comerciales. Las consideraciones de responsabilidad social permanecen esencialmente dentro de la esfera privada.

Por otro lado, es paradójico que los empleados no toman consciencia en forma espontánea de esta propuesta de valor que les concierne directamente: la consideración de los aspectos sociales por parte de la empresa. En otras palabras, el hecho de querer mantener a toda costa una parte de su producción en el territorio y generar empleo local es una opción económica difícil para un dirigente. Aunque esta elección ayude a los empleados, en realidad esta no es percibida por ellos. Esto lo evidencian las siguientes palabras, que demuestran que el dirigente intenta hacerles ver el esfuerzo en favor del empleo:

Traemos a mucha gente aquí; personalidades del mundo de los negocios, del mundo político de todas las tendencias, locales, regionales, etc... Traemos gente para sensibilizarla. Hemos pegado la etiqueta OFG por todas partes. Es importante que nuestros empleados tomen conciencia (...). Lo que ellos ven, es que estamos luchando, esta- 
mos tratando de seguir apostando a seguir fabricando en Francia... Es una herramienta de comunicación. ¡No puedo decir que ellos son quienes nos impulsan para hacer esto! ¡No! Esto es una decisión del dirigente.

Desde luego, el cliente no es el único interesado por la oferta y la propuesta de valor de la empresa. Las autoridades locales parecen estar especialmente atentas al hecho de que una empresa participe en el desarrollo de su territorio. Por lo tanto, la dimensión económica y social de la propuesta de valor (la creación de riqueza, mantenimiento y desarrollo del empleo en el territorio) es esencial en este sentido. La principal razón es clara: los recursos de las autoridades están directamente relacionados con la tributación de las empresas y los individuos. Este es un gran desafío, sobre todo en un contexto de disminución de los subsidios estatales. De acuerdo con ello, las autoridades locales toman en cuenta a las empresas que intervienen en el juego de su territorio, incluso a través de sus propios medios de comunicación (la página web, local, mensual...). Subráyese que se trata también de una cuestión de marketing territorial, la presencia de empresas faro puede tener un efecto positivo en la aparición o la llegada de otros jugadores propensos a impulsar la economía local.

Por último, se analiza el polo del modelo RCOV, el cual se refiere a la organización de la cadena y de la red de valor de la empresa. En cuanto al anclaje territorial, dos impactos importantes se pueden señalar: el primero se refiere a la recomposición de la cadena y el valor de la red de negocios; el segundo a la importancia tomada por las principales actividades de apoyo consideradas, tomando en cuenta el posicionamiento de alta gama y la etiqueta OFG.

Recuérdese que la etiqueta OFG establece que el $50 \%$ del valor agregado (VA) debe realizarse en el suelo francés, es decir, el producto toma sus principales características en Francia. Obviamente, la etiqueta OFG es un incentivo para buscar proveedores de preferencia en ese país. Sin embargo, esto a veces no es posible, ya que en ocasiones no existen los proveedores de ciertos productos, componentes o materiales en el territorio nacional (por ejemplo, el caso del acero inoxidable). Esa problemática leva finalmente a los administradores encargados de las compras a plantearse con claridad la cuestión del sourcing.

Con relación al aprovisionamiento, el temor de los dirigentes de la empresa es, por lo general, el costo de materiales o componentes comprados en Francia. Sin embargo, creer que un proveedor francés es sistemáticamente más caro que otro extranjero sería un error. Uno de los dirigentes relata lo siguiente:

Buscando, me encontré con un proveedor con el que trabajé hará alrededor de quince años. Me dijo -lo recuerdo muy bien-: "Hemos perdido el mercado" (...). El contacto se detuvo, no sabemos por qué. Yo le dije: "¿Usted podría hacerme algunas cotizaciones? Estoy interesado en volver a trabajar con usted". Él me dio sus precios más baratos que mi actual proveedor español. Si yo no tuviera la exigencia de la OFG me habría quedado con el otro... Restablecimos nuestro mercado...

Finalmente, ya es posible observar que el enfoque del anclaje territorial conduce a la empresa a rexaminar su cartera de proveedores. Si esto puede conducirle al cuestionamiento de las relaciones a largo plazo con un comprador extranjero, es igualmente dentro de una óptica de colaboración que la empresa busca realizar sus relaciones con un nuevo proveedor francés.

Según el dirigente entrevistado, el proceso de obtención de la etiqueta OFG no lleva a la empresa a internalizar las actividades subcontratadas originalmente. Dicho esto, para definir su política industrial, la empresa reconoce implícitamente 
esta presión del valor agregado (VA) que debe llevarse a cabo en Francia en el lanzamiento de nuevos productos. En esta etapa, es necesario disociar lo que pertenece a la estrategia y lo que no: algunos componentes no están etiquetados (por ejemplo, la perilla de intensidad de gas de encendido del calentador). Mientras que otros son el resultado de know-how raros (por ejemplo, el sistema de energía en la plancha de cocción de gas). En cuanto a la primera, la empresa puede internalizar las actividades en la medida en que tiene el know-how correspondiente, o buscar un proveedor francés, si los hubiere, o buscar en el extranjero. El arbitraje se basa en tres parámetros: el nivel general de VA alcanzado en el producto final (50\% mínimo "francés"), el costo de fabricación o suministro componente y la distribución de cargas para los talleres. Así, la empresa opta por internalizar la producción de un componente que le permite desacelerar su actividad durante los períodos de baja.

Sin embargo, en el conjunto de la producción de la empresa, dos tipos de componentes (placa de la plancha de cocción y sistema de suministro de gas) pertenecen a know-how raros: el esmaltado de la plancha de cocción y la fabricación de sistemas de suministro de gas (este último necesita obtener la aprobación de un organismo certificado). El primero, se posee a lo interno; este permite a la empresa ofrecer productos que realmente la diferencian de sus competidores y, en esencia, gran parte del valor agregado (VA) por la empresa es resultante de esa operación de producción. La segunda no es poseída más que por unos pocos fabricantes franceses o extranjeros. Por lo tanto, la empresa no tiene otra alternativa que externalizar una parte de su producción. Tratándose de los componentes estratégicos, además de la cuestión de los costos de suministro, la calidad es un factor esencial para la elección del proveedor. En este caso, este segundo elemento lleva a la empresa a su vez a optar por un fabricante italiano, que ofrece un mayor nivel de calidad que los otros proveedores potenciales.

La importancia de las actividades de apoyo es también una de las principales consecuencias del enfoque de anclaje territorial. Tres actividades son especialmente impactadas: 1) el desarrollo tecnológico y la investigación-desarrollo, 2) los recursos humanos y 3) las compras y el aprovisionamiento.

La OFG, como se ha mencionado anteriormente, es el resultado de un posicionamiento de alta gama (PAG). Esta es a la vez un símbolo de la calidad del producto y una defensa de ciertos valores de la empresa. Tomando eso en cuenta, la diferencia de precio entre un bien fabricado predominantemente en Francia y otro producto proveniente de un país con bajos costos de mano de obra (por ejemplo China), requiere otros elementos de diferenciación. En efecto, la empresa debe hacer todo lo posible para evitar la banalización de sus productos. Esta es la razón por la cual la innovación está en el corazón de la problemática de la empresa ENO, que desea continuar desarrollándose en Francia y proyectarse al extranjero. Los resultados también son convincentes, puesto que ENO es reconocida por ser una empresa innovadora (cf. supra).

En ese sentido, el proceso de innovación tiene que adaptarse al know-how (le savoir-faire) histórico (por ejemplo, el esmaltado) para evitar que la empresa sea penalizada en términos de sus costos y así cumplir con las exigencias de calidad de la OFG. Es por tal razón que la empresa abandona la técnica artesanal (manual) de aplicación de pintura de esmalte por la aplicación que utiliza la robótica. El desafío es poner esta "a la moda" en el sistema de fabricación, adaptándola a las necesidades actuales.

El impacto es obviamente notable en los recursos humanos, ya que la industrialización de los productos requiere ahora la intervención de los ingenieros, en donde antaño el técnico ocupaba 
el lugar del obrero. Así, se observa en el seno de la empresa el aumento de competencias y el paso de una cultura tradicional basada en el know-how (le savoir-faire) a una cultura industrial basada en el know-how tecnológico. La empresa responde a ese desafío mediante la contratación de jóvenes a través de dispositivos alternos. Estos nuevos obreros son capacitados dentro de la empresa, estos aportan su conocimiento sobre las tecnologías actuales, permitiendo la transformación del know-how artesanal en know-how industrial (externalización). En este sentido, se trata de la socialización en la espiral del conocimiento, como lo establece Nonaka (1994).

En este caso, la inserción de los jóvenes permitirá a la empresa promover las combinaciones de conocimiento explícito, la redacción de manuales que constituyen, en la terminología de Nonaka, uno de los cuatro modos de creación de conocimiento en la empresa. Así, el impacto del anclaje territorial en la gestión de recursos humanos es fundamental, pues la cuestión es la del aprendizaje organizacional.

La tercera actividad impactada por el enfoque del anclaje territorial es la compra y el suministro. El problema del comprador gira en torno a tres criterios que son el costo de compra de bienes o componentes, la calidad de suministro y el tiempo de entrega, incluyendo el respeto de este. Una variable adicional que se conjuga -reitérese- es el porcentaje del total de valor agregado (VA) francés, sobre el VA total realizado en el producto. Si el nivel de 50\% no es alcanzado, el comprador deberá necesariamente encontrar un proveedor en suelo francés, esto independientemente de los costos potenciales. Esto requerirá un esfuerzo de prospección por parte de la empresa, pero también, en algunos casos, el establecimiento de una política de asociación con los proveedores franceses para asistirlos en la creación de un nuevo canal de suministro franco-francés. En otras palabras, el papel de la empresa como comprador está menos en una lógica transaccional y más en una asociación frente a sus proveedores, a los cuales esta necesita para obtener la etiqueta OFG.

\section{RESULTADOS Y CONCLUSIONES}

El principal interés del modelo de negocios (MN) como concepto, es centrar la problemática de la empresa sobre el tema de la creación de valor. Sin embargo, la idea misma de "creación de valor" es ambigua. Esta se refiere a dos preguntas fundamentales: ¿De qué valor se habla específicamente? ¿A quién está destinada esa creación de valor? En la visión clásica, esas cuestiones se omiten: se trata, obviamente, del valor económico creado por la empresa y el financiero para los accionistas. Pero en la visión asociativa de la empresa (Charreaux, 2002), que parece prevalecer en la actualidad, se debe extender esa cuestión de la creación de valor a su dimensión no económica y ampliar el alcance de sus beneficiarios (accionistas y grupos de interés, shareholders y stakeholders).

Frente al nomadismo económico y los excesos generados por el fenómeno de la globalización, muchas empresas optan por establecer un compromiso en su territorio. Sin embargo, muchas razones pueden ahora incitarlas a abandonar su lugar de implantación: por ejemplo, menores costos de producción por la implementación en un país con un bajo costo de mano de obra, fácil acceso a los recursos o cierto know-how, o la integración en una organización industrial mundial. Sin embargo, el anclaje territorial, sin ser obviamente desinteresado, cumple con la disposición de una empresa para contribuir al desarrollo del territorio que la acoge en una lógica de "ganar-ganar". De hecho, eso demuestra la preocupación del dirigente no solo para equilibrar los intereses en juego (económicos versus sociales), sino también para ampliar el alcance de los beneficiarios del valor creado por la empresa (accionistas versus grupos de interés). 
El objetivo de este trabajo, recuérdese, ha sido ilustrar a través de un estudio de caso las consecuencias del enfoque del anclaje territorial en el modelo de negocios de una empresa. Para ese propósito, se utiliza el modelo RCOV de Lecoca et al. (2006); este describe las tres dimensiones principales sobre los cuales se basan las decisiones de la empresa para generar ingresos, a saber: los recursos y las competencias movilizadas, la oferta a los clientes (entendido en el sentido más amplio), la organización interna de la empresa y sus transacciones con socios externos.

Con respecto al componente recursos y competencias (RC), el impacto del anclaje territorial es significativo en cada uno de los principales recursos movilizados por la empresa. En general, existe una elevación de la gama que obliga a la empresa a invertir en tecnología (producción robótica) que la hace más eficiente. Esto, dicho sea de paso, requiere dedicar recursos financieros. La innovación tecnológica conduce a un cambio en la política de recursos humanos para el paso de una lógica artesanal a una lógica info-industrial de producción. Consecuencia de estas transformaciones técnico-administrativas, es la decisión de la gerencia para obtener un reconocimiento por sus esfuerzos en el anclaje territorial con una etiqueta específica: Origine France Garantie (OFG). Si las competencias iniciales de la empresa (en este caso el know-how del esmalte de hierro fundido y acero) le permiten visionar el anclaje de sus actividades en su territorio histórico, el enfoque del anclaje territorial le obliga a adaptar sus competencias iniciales a las necesidades actuales, pasando de una forma de producción artesanal a una info-industrial. El desafío para el caso de ENO ha sido cristalizar el know-how de los operadores en un verdadero aprendizaje organizacional.

En cuanto a la segunda parte, relativa a la organización de la cadena y de la red de valor, el anclaje territorial conduce a abordar claramente el problema del sourcing. La empresa no podría postular un enfoque de anclaje territorial y practicar al mismo tiempo una política de compras ligada a una forma de nomadismo en relación con sus propios aprovisionamientos. En ese nivel de análisis existe una verdadera obligación tomando en cuenta la etiqueta Origine France Garatie (OFG) obtenida por ENO (50\% del VA debe provenir de Francia). Por lo tanto, la empresa está obligada a reexaminar su cartera de proveedores y considerar nuevas asociaciones con proveedores franceses que tenía y que habían sido previamente rechazadas por razones de costos.

En esta etapa también es posible interrogarse sobre la posibilidad de algún aprovisionamiento francés. La desaparición de determinados know-how hace imposible, a veces, considerar un aprovisionamiento francés (por ejemplo, el caso del acero inoxidable). En otras palabras, el anclaje territorial tal vez sea afectado por la falta de un aprovisionamiento en el territorio. Otro punto significativo del anclaje territorial es el reforzamiento de las actividades: fuerte énfasis en la actividad de investigación y desarrollo (ID) e innovación, debido al posicionamiento de productos y una voluntad de diferenciación notable en relación con la competencia.

Por último, el tercer componente es el valor creado por la empresa. Con respecto a ello, el anclaje territorial responde más o menos claramente a las peticiones de los grupos de interés (stakeholders). La oferta y la propuesta de valor encuentra una respuesta positiva en el mercado no solo porque brinda seguridad a los clientes ("este producto, sabemos dónde se fabricó...", "es un producto de calidad"), sino también porque les permite influir en lo que parecía ser una fatalidad desde hace mucho tiempo: el fenómeno la desindustrialización en Francia. La comunicación de la empresa, cualquiera que sea su naturaleza institucional o comercial, debe centrarse tanto en criterios comerciales como cualitativos y simbólicos. Al contrario, hay que admitir que, a parte de las autoridades locales interesadas en la 
afirmación de un anclaje territorial de las empresas que operan en su área geográfica (recursos financieros inducidos, elemento del marketing territorial), la propuesta de valor, especialmente el no económico, encuentra poco eco en otros grupos de interés (stakeholders).

\section{REFERENCIAS}

Brundtland, R. (1987). Notre avenir à tous, Recuperado de: https://www.diplomatie.gouv.fr/sites/odyssee-developpement-durable/files/5/rapport_ brundtland.pdf

Cariou, Y., Fournie, S. y Wallet F. (2006). Le bilan sociétal: un outil de management pour renforcer l'ancrage territorial et la responsabilité sociale des coopératives agricoles. Revue Développement durable \& territoires, dossier 5. doi: 10.4000/developpementdurable.1626

Charreaux, G. (2002). À la recherche de nouvelles fondations pour la finance et la gouvernance d'entreprise. Finance Contrôle Stratégie, 5(3), 5-68.

Chesbrough, H. y Rosenbloom, R.S. (2002). The Role of the Business Model in Capturing Value from Innovation: Evidence from Xerox Corporation's Technology Spinoff Companies. Harvard Business School, 01-002.

Dahan, N.M., Doh, J.P., Oetzel, J. y Yaziji, M. (2010). Corporate-NGO Collaboration: Co-Creating New Business Models for Developing Markets, Long Range Planning, 43(2-3), 172-194.

Demil, B. y Lecocq, X. (2010). Business Model Evolution: In Search of Dynamic Consistency, Long Range Planning, 43, 227-246.

Hadly-Rispal, M. (2002). La méthode des cas. Application à la recherche en gestion, France: Ed. De Boëck Université.

Harribey, L. y Cardebat, J. (2013). L'évolution de la perception du développement territorial durable dans les PME françaises permet-elle un renouvellement de la gouvernance territoriale? Une réponse à partir de deux enquêtes. Mana- gement \& Avenir, 59(1), 102-119. doi:10.3917/ mav.059.0102.

Le Gall, S. y Bériot, D. (2013). Orienter la stratégie de l'entreprise vers le territoire: une recherche action par une approche systémique, Vie \& sciences de l'entreprise, 194, 27-47.

Lecocq, X., Demil, B. y Warnier, V. (2006). Le business model, un outil d'analyse stratégique, L'Expansion Management Review, 123, hiver, 96-109.

Marx, K. (1965) Le capital, livre I, Paris: NRF Gallimard.

Maucuer, R. (2013). Partenariats ONG-entreprise et évolution du business model de la grande entreprise. Le cas de Suez-Environnement, Thèse de Doctorat, Université Paris-Dauphine, France.

Moingeon, B. y Lehmann-Ortega, L. (2010). Genèse et Déploiement d'un Nouveau Business Model: l'Etude d'un Cas Désarmant, M@n@gement, 4, 266-297.

Nonaka, I. (1994). A dynamic theory of organizational knowledge creation. Organization Science, 1(5), 14-36.

ORSE (2006). L'ancrage des entreprises dans les territoires: pilotage et reporting international, IMS Entreprendre pour la Cité.

Osterwalder, A. y Pigneur, Y. (2009). Business Model Generation, USA:Tim Clark Edition.

Osterwalder, A., Pigneur,Y. y Tucci, C.L. (2005). Clarifying Business Models: Origins, Presents, and Future of the Concept, Communications of AIS, 15, Article 1.

Porter, M. E. (1985). The Competitive Advantage: Creating and Sustaining Superior Performance. NY: Free Press.

Porter, M.E. y Kramer, M.R. (2011). Creating Shared Value. How to Reinvent capitalism and unleasch a wave of innovation and Growth. Harvard Business Review, 89, enero-febrero, 62-77.

Stähler, P. (2002). Business Models as an Unit of Analysis for Strategizing Proceedings of the Internatio- 
nal Workshop on Business Models, Switzerland, Lausanne, 4-5 de octubre.

Stake, R.E. (1995). The Art of Case Study Research. USA:Thousand Oaks, CA: Sage Publication.

Stewart, D.W. y Zhao, Q. (2000). Internet marketing, business models, and public policy, Journal of Public Policy. Cambridge, 19 (Fall), 287-296.

Tikkanen, H., Lamberg, J.A., Parvinen, P. y Kallunki, J.P. (2005) Managerial cognition, action and the business model of the firm, Management Decision, 6(43), 789-809.

Vial, V. (2008). How Socially Responsible Engagement Can Change Your Business Model: The Radical Experience of Armor Lux, Global Business and Organizational Excellence, noviembre-diciembre, 24-34.

Yin, R. K. (1990). Case Study Research: Design and Methods, Applied Social Research Methods series. USA, 5, CA: Sage, Newbury Park.
Yunus, M., Moingeon, B. y Lehmann-Ortega L. (2010). Building Social Business Models: Lessons from the Grameen Experience. Long Range Planning, 43, 308-325.

Zimmermann, J.B. (2005). Entreprises et territoires: entre nomadisme et ancrage territorial. Revue de l'IRES, 47, 21-36.

Zimmermann, J.B. (2008). Le territoire dans l'analyse économique. Proximité géographique et proximité organisée. Revue Française de Gestion, 184, 105-118.

Zott, C. y Amit, R. (2010). Business Model Design: An Activity Perspective, Long Range Planning, 43, 216-226.

Recibido 10 de mayo 2017

Aceptado 15 de julio 2017 
This document was created with Win2PDF available at http://www.win2pdf.com. The unregistered version of Win2PDF is for evaluation or non-commercial use only. This page will not be added after purchasing Win2PDF. 